\title{
REA_02 - A new multi-species Protein A-ELISA assay for plague diagnosis in humans and other mammal hosts
}

Matheus Filgueira Bezerra ${ }^{1 *}$; Camila Cavalcanti Xavier ${ }^{1}$; Alzira Maria Paiva de Almeida $^{1}$; Christian Robson de Souza Reis ${ }^{1}$.

${ }^{1}$ Instituto Aggeu Magalhães Fiocruz/PE.

Introduction: The Hemagglutination assay (HA) has been used for several decades in the diagnosis of bubonic plague. Although useful, this technique presents negative aspects, such as inter-observer interpretation bias, high consumption of F1 antigen and risk of cross-reaction with other pathogens. The development of new immunoenzymatic tests to replace HA brought significant improvements in plague serological diagnosis, however, conventional ELISA requires specific conjugates for each species, which is frequently a setback for plague surveillance, considering the wide range of plague natural hosts.

Objective: To overcome these limitations, we aimed to develop and validate a new indirect ELISA method using a Protein A-peroxidase conjugate to detect anti-F1 antibodies across several mammal species, including humans.

Methodology: Distinct F1 antigen concentrations and serum/conjugate dilutions were tested to establish optimal conditions. To determine the cut-off and performance rates, 288 samples ( 81 control rabbits, 64 humans, 66 rodents and 77 dogs) from the Brazilian Plague Reference Service were characterized as positive (98) or negative (190) according to HA. Additionally, these samples were also tested for IgGELISA, using a distinct IgG conjugate for each group. Next, to evaluate the agreement rate between conventional IgG and the new Protein A-ELISA methods, we tested 487 sera for both methods, using the previously established cut-offs.

Results: We found optimal Protein A-ELISA conditions using $250 \mathrm{ng}$ of F1 antigen per well, a 1:500 dilution for testing sera and 1:10.000 for Protein A-peroxidade conjugate. Of note, when tested with sera from rabbits exposed to Yersinia enterocolitica $(\mathrm{n}=2)$ and Yersinia pseudotuberculosis $(\mathrm{n}=5)$, there was no significant cross-reaction. Using HA as reference, the overall sensitivity/specificity and Kappa index of Protein A-ELISA was 93.9/98.9 and 0.938 (97.3/97.7 and 0.950 for rabbits; 100/100 and 1.0 for humans; 80/100 and 0.848 for rodents and 95.0/98.2 and 0.932 for dogs) respectively. The Receiver Operating Characteristic (ROC) Curve showed an area under of curve (AUC) of 0.993 in overall analysis with similar results for each species. Similar or slightly inferior results were observed for the IgG-ELISA protocol, with sensitivity/specificity, Kappa index and AUC of 97.3/100, 0.975 and 0.974 for rabbits; $85.7 / 100,0.851$ and 0.930 for humans and 90.0/100, 0.930 and 0.982 for dogs, respectively. Moreover, the positive/negative OD ratios were higher in Protein A-ELISA than in IgG-ELISA (81.1 versus 22.4 for rabbits, 34.3 versus 11.8 for humans and 12.8 versus 4.85 for dogs). Next, by testing both the Protein A and IgG conjugates protocols for 487 sera not previously tested for HA, a strong agreement was observed (kappa $=0.973)$.

Conclusion: In summary, the Protein A-ELISA method here described showed high performance when compared both to the hemagglutination and the conventional IgG-ELISA, with a single protocol that is polyvalent to the main natural plague hosts and requires reduced amounts of F1 antigen used per test.

Keywords: ELISA; Protein A; Bubonic plague 